Journal of Computer Science 7 (6): 821-822, 2011

ISSN 1549-3636

(c) 2011 Science Publications

\title{
Comment on "Method of Fingerprint Identification"
}

\author{
Ford Lumban Gaol \\ Faculty of Graduate Program Information Technology \\ Swiss German University, Indonesia
}

\begin{abstract}
Problem statement: Paper that written by Surachai Panich reported matching two fingerprints taken from small database of fingerprints. Approach: In this study we used extensive literature review and research publication. We also compare with some best practices that applied in the business and industrial field for matching the fingerprints. Results: Surachai Panich used his method only for around one hundred data. However, we found that in the recent research most of the fingerprint problems are explore in million of data. I found some of the literature review are quiet old. Since the advancement of Fingerprint matching is increase very rapidly so the comparison with the recent publication is a must. The comparison has to be in the experimental using million of data as the input. We do not find such experimental result on this paper. Conclusion/Recommendations: The paper should explore more in biometrics feature extraction for matching process. The biometrics have analogy to the use of physiological or biological characteristics to measure the identity of an individual. These features are assuming unique to each individual and remain unaltered during a person's lifetime. These features make biometrics a promising solution to the society.
\end{abstract}

Key words: Fingerprint matching, fingerprint identification, biometric system

\section{INTRODUCTION}

Panich (2010) reported about finding out an effective algorithm in order to match two fingerprints taken from database. This study state that fingerprint identification techniques only used in a small database in order to find out an effective algorithm to develop the accuracy in matching process. However I found that in the real application, the fingerprint matching must be in very large database. I argue that in the recent research most of the fingerprint problems are explore in million of data. Biometrics have analogy to the use of physiological or biological characteristics to measure the identity of an individual. These features are assuming unique to each individual and remain unaltered during a person's lifetime. These features make biometrics a promising solution to the society. In traditional access control, the access to the secured area can be made by the use of ID numbers or password which amounts to knowledge based security. But such mode can easily be modified and penetrated by intruders and they can breach the doors of security. In monetary transactions and highly restricted to information zone, these are very serious situation. Thus to overcome the above mentioned issue biometric traits are used (Alfaouri, 2008).

The various biometrics traits available are face, fingerprint, iris, palm print, hand geometry and ear.
Among the available biometric traits some of the traits outperform others. The reliability of several biometrics traits is measured with the help of experimental results (Panich, 2010; Khairul and Osamu, 2010).

The biometric system is basically divided into two modes i.e., unimodal biometric system and multimodal biometric system. In case of unimodal biometric system the individual trait is used for recognition or identification. The most successful applications of image analysis and understanding, face recognition has recently received significant attention, especially during the past several years, for example, recognition of face images acquired in an outdoor environment with changes in illumination and/or pose remains a largely unsolved problem. In other words, current systems are still far away from the capability of the human perception system, research in biometric systems has been increasing significantly due to international insecurity environment. Research groups around the world are developing algorithm and systems based on face, iris, fingerprint, palm print or voice and one other possible biometric source is the ear (Sayeed et al., 2009; Rizon et al., 2006; Galbiati, 1990; Hong et al., 1998; Rehan and Rashid, 2004; Khairul and Osamu, 2010; Tabassi and Wilson, 2005).

Paper that written by Panich (2010) reported about matching two fingerprints taken from small database of 
fingerprints. Regarding with Surachai Panich, he used his method only for around one hundred data.

The references also are quiet old since the most recent reference is year 2004. The author also did not mention about the algorithm that he proposed to use.

I found that the author also has to compare with some recent paper that published recently that can be explored to enhance the state of the art of the study such (Yuen et al., 2009; Ross et al., 2006, Xinjian et al., 2006; Sharat et al., 2005).

On Part Materials and Method, Panich (2010) also mentioned that the proposed fingerprint identification system focused on four main tasks. But I found on page 1062 only listed three task.

Base on the work of (Gille, 2005; Feng, 2008), the most important part if we work with Fingerprint Identification using large database is the data structured. We have to define effective and efficient data structured that transform from images of database. Surachai Panich did not mention about that in his study.

On the part of the Result, I do not find any experimental result as mention on the abstract. It should be put the performance of the algorithm and compare with other Fingerprint Matching algorithm. Some of the novel fingerprint matching algorithm can also found in (Greenberg et al., 2000; Xinjian et al., 2006; Sharat et al., 2005).

Panich (2010) mentioned on the conclusion that the algorithm reached high accuracy when perform on small database of 100 fingerprint images. I do not find any experimental result for the accuracy experiment. I suggest that the author has not suppose mention it on the Conclusion.

\section{REFERENCES}

Greenberg, S., M. Aladjem and D. Kogan, 2000. Fingerprint image enhancement using filtering techniques. Real-Time Imag., 8: 227-236. DOI: 10.1006/rtim.2001.0283

Galbiati, L., 1990. Machine Vision and Digital Image Processing Fundamentals. Facsimile Edn., Prentice-Hall, Eaglewood Cliffs, New Jersey, ISBN: 10: 013542044X, pp: 180.

Hong, L., Y. Wan and A. Jain, 1998. Fingerprint image enhancement: Algorithm and performance evaluation. IEEE Trans. Patt. Anal. Mach. Intell., 20: 777-789. DOI: 10.1109/34.709565

Rehan, N. and K. Rashid, 2004. Multi-matcher based fingerprint identification system. J. Applied Sci., 4: 611-618. http://scialert.net/pdfs/jas/2004/611618.pdf
Khairul, H.A. and O. Osamu, 2010. Implementation of multi-centroid moment invariants in thermal-based face identification system. Am. J. Applied Sci., 7: 283-289. DOI: 10.3844/ajassp.2010.283.289

Ross, A., J. Reisman and A. Jain, 2006. Fingerprint matching using feature space correlation. Lecture Notes Comput. Sci., 2359: 48-57. DOI: 10.1007/3540-47917-1_6

Feng, J., 2008. Combining minutiae descriptors for fingerprint matching. Patt. Recog., 41: 342-352. DOI: 10.1016/j.patcog.2007.04.016

Xinjian, C., T. Jie and Y. Xin, 2006. A new algorithm for distorted fingerprints matching based on normalized fuzzy similarity measure. Image Process. IEEE Trans., 15: 767-776. DOI: 10.1109/TIP.2005.860597

Sharat, C., A. Cartwright and V. Govindaraju, 2005. Kplet and coupled BFS: A graph based fingerprint representation and matching algorithm. Adv. Biometrics Lecture Notes Comput. Sci., 3832: 309315. DOI: 10.1007/11608288_42

Tabassi, E. and C.L. Wilson, 2005. A novel approach to fingerprint image quality. Image Process., 37-45.

Alfaouri, M., 2008. Image recognition using combination of discrete multi_wavelet and wavenet transform. Am. J. Applied Sci., 5: 418-426. DOI: 10.3844/ajassp.418.426

Rizon, M., M. Hashim, P. Saad and S. Yaacob, 2006. Face recognition using eigenfaces and neural networks. Am. J. Applied Sci., 2: 1872-1875. DOI: 10.3844/ajassp.2006.1872.1875

Sayeed, S., N.S. Kamel and R. Besar, 2009. A novel approach to dynamic signature verification using sensor-based data glove. Am. J. Applied Sci., 6: 233-240. DOI: 10.3844/ajassp.2009.233.240

Yuen, C.T., M. Rizon, W.S. San and T.C. Seong, 2009. Facial features for template matching based face recognition. Am. J. Applied Sci., 6: 1897-1901. DOI: 10.3844/ajassp.2009.1897.1901

Gille, W., 2005. Analysis of the chord length distribution along an edge: The wedge case and the quadratic rod case. J. Math. Stat., 1: 106-112. DOI: 10.3844/jmssp.2005.106.112

Panich, S., 2010. Method of fingerprint identification. J. Comput. Sci., 6: 1062-1064. DOI: 10.3844/jcssp.2010.1062.1064 